\title{
Towards Teaching by Demonstration for Robot-Assisted Minimally Invasive Surgery
}

\author{
Hang $\mathrm{Su}^{1}$, Member, IEEE, Andrea Mariani ${ }^{2}$, Salih Ertug Ovur ${ }^{1}$, Arianna Menciassi ${ }^{2}$, Senior Member, IEEE, \\ Giancarlo Ferrigno $^{1}$, Senior Member, IEEE, and Elena De Momi ${ }^{1}$, Senior Member, IEEE
}

\begin{abstract}
Learning manipulation skills from open surgery provides more flexible access to the organ targets in the abdomen cavity and this could make the surgical robot working in a highly intelligent and friendly manner. Teaching by Demonstration (TbD) is capable of transferring the manipulation skills from human to humanoid robots by employing active learning of multiple demonstrated tasks. This work aims to transfer motion skills from multiple human demonstrations in open surgery to robot manipulators in Robot-Assisted Minimally Invasive Surgery (RAMIS) by using TbD. However, the kinematic constraint should be respected during the performing of the learned skills by using a robot for Minimally Invasive Surgery. In this paper, we propose a novel methodology by integrating the cognitive learning techniques and the developed control techniques, allowing the robot to be highly intelligent to learn senior surgeons' skills and to perform the learned surgical operations in semi-autonomous surgery in the future. Finally, experiments are performed to verify the efficiency of the proposed strategy and the results demonstrate the ability of the system to transfer human manipulation skills to a robot in RA-MIS and also shows that the remote center of motion ( $\mathrm{RCM})$ constraint can be guaranteed simultaneously.
\end{abstract}

Note to Practitioners-This paper is inspired by limited access to the manipulation of laparoscopic surgery under a kinematic constraint at the point of incision. Current commercial surgical robots are mostly operated by teleoperation, which is representing less autonomy on surgery. Assisting and enhancing the surgeon's performance by increasing the autonomy of surgical robots has fundamental importance. The technique of $\mathrm{TbD}$ is capable of transferring the manipulation skills from human to humanoid robots by employing active learning of multiple demonstrated tasks. With the improved ability to interact with humans, such as flexibility and compliance, the new generation of serial robots become more and more popular in nonclinical research. Thus, advanced control strategies are required by integrating cognitive functions and learning techniques into the processes of surgical operation between robots, surgeon and MIS. In this article, we propose a novel methodology to model the manipulation skill from multiple demonstrations and execute the learned operations in RA-MIS by using a decoupled controller to respect the RCM constraint exploiting the redundancy of the robot. The developed control scheme has the following functionalities: 1) it enables the 3-D manipulation skill modeling after multiple demonstrations of the surgical tasks in open surgery by integrating DTW and GMM based DMP. 2) it maintains the RCM constraint in a smaller safe area while performing the learned operation in RA-

*This work was supported by the European Commission Horizon 2020 research and innovation program, under the project SMARTsurg, grant agreement No. 732515. (Corresponding author: Hang Su.)

${ }^{1}$ Hang Su, Salih Ertug Ovur, Giancarlo Ferrigno and Elena De Momi are with the Dipartimento di Elettronica, Informazione e Bioingegneria, Politecnico di Milano, 20133, Milano, Italy. (e-mail: hang.su@ polimi.it; salihertug.ovur@polimi.it; giancarlo.ferrigno@polimi.it; elena.demomi@polimi.it).

${ }^{2}$ Andrea Mariani and Arianna Menciassi are with The BioRobotics Institute, Scuola Superiore Sant'Anna, 56127 Pisa, Italy. (e-mail: andrea.mariani@santannapisa.it; arianna.menciassi@santannapisa.it).
MIS. The developed control strategy can also be potentially used in other industrial applications with a similar scenario.

Index Terms-Teaching by Demonstration, Dynamic Time Warping, Dynamic Movement Primitive, Remote Center of Motion, Robot-Assisted Minimally Invasive Surgery

\section{INTRODUCTION}

$\mathbf{R}$ OBOT-ASSISTED Minimally Invasive Surgery (RAMIS) has become more popular over recent years because of the benefits in advanced surgical precision, increased movement range, improved proficiency, and enhanced vision for surgeons [1], [2], [3]. Compared with the traditional open surgery method, MIS can minimize the scale of the wound on the patients' body and further avoid causing damage to the surrounding organs ad tissues. Benefit from this, the patient's recovery time after surgery can be significantly shortened, further reducing the patient's pain. However, there are several critical issues needs to be considered. Firstly,the manipulation features the surgical tool going through small abdominal incisions with lengths less than an inch on the abdominal wall in Minimally Invasive Surgery (MIS) [4], known as the remote center of motion (RCM) limitation, resulting in a kinematic constraint of surgical robot when conducting surgical operations [5]. Compared to conventional open surgical procedures, intensive training is required to train a novice surgeon to perform MIS operations. Due to the complexity of the skills in kinematic constraints, it allows an intuitive access to surgical operations [6], [7]. In fact, the movement of a surgical instrument is mirrored to the opposite way inside the patient under the RCM constraint, as well as the applied force depends on the distance from the entry point, which is known as the "fulcrum effect".Hence, to ensure the safety during surgical operation, the movement of the surgical tool should be constraint.

Furthermore, current commercialized surgical robots are simply controlled by surgeons using teleoperation, and they involve less autonomy in the surgical operation [8]. It is a of great significance to assist and enhance the performance of surgeons by increasing the autonomy of surgical robots. Increasing the autonomy of surgical robots when conducting such particular complex surgical operations, such as suturing or knotting, can potentially reduce the length of surgical procedures and reduce the workloads of surgeons to avoid fatigue [9], [10], [11], [12], as well as improving tracking accuracy with the development of technology in artificial intelligence and cognition progress. In the past decade, the 
developed commercial medical systems which incorporate autonomous and semi-autonomous technologies, as well as experimental work on the automation of numerous surgical procedures, have attracted much research interests [8]. In order to meet this demand, it is necessary to transfer the manipulation skills from human to surgical robots after showing the correct execution of the trajectories. This approach is known as Teaching by Demonstration (TbD) [13]. Calinon et al. [14] had investigated how human motor skills can be applied to the robot. Yang et al. [15] developed an interface in the human-robot communication framework for humanimpedance adaptive skill transfer. Takayuki et al. developed an automated knot tying system that can learn to tie knots after just one demonstration done by a surgeonn[16]. However, a single demonstration is lack of consistency, and they are not enough to model a good manipulation skill library. Hence, multiple demonstrations are essential for the extraction of the manipulation skills and training of the skill model. For example, in [17], Petitjean et al. adopted the time regular function to describe their time correspondence and to calculate the minimum distance among the test and reference template to describe their time correspondence. Kormushev et al. [18] studied the comprehension of the trajectory design for the spherical obstacle by using Dynamic Motion Primitives (DMP) modeling combined with the synthetic capacity discipline method. A learning framework for human-to-robot adaptive manipulation skills was developed in [19]. Li et al. [20] presented methodology to learn 2-D drawing skill from multiple demonstrations.

During MIS surgical operation process, in addition to human motion skill learning and transferring to the surgical robot, the RCM constraint on the abdominal wall of the patients' body [21] has to be respected [22] simultaneously. In general, the RCM constraint can work actively or passively. The passive constraint is physically enforced, while a software supported controller needs to be considered for active constraints [23]. Since specialized surgical robots with passive RCM constraints are expensive; therefore, their usage in hospitals is limited. Different approaches have been introduced to solve the RCM as a kinematic constraint [24]. Using serial robots and achieving the RCM constraint with their redundancy [25], [26], [27] is cost-effective and offers versatile workspace, which has a high interest in the medical field, in particular for MIS. In our previous works [23], [28], the RCM constraint had been solved in a decoupled way by exploiting the redundancy of the robot, and the controller had shown prominent performance to guarantee the RCM constraint without any influence on the surgical tooltip. It delivers the surgical robot operating in a highly intelligent and friendly manner.

In this paper, a novel methodology by integrating cognitive functions and learning techniques is considered to the processes of surgical operation between robots, surgeon, and MIS. Thanks to the proposed approach, manipulation skill can be learned from multiple demonstrations and the learned operations can be performed in RA-MIS by using a decoupled controller to respect the RCM constraint by exploiting the redundancy of the robot. Moreover, Dynamic Time Warping (DTW) is adopted to process the data acquired from the
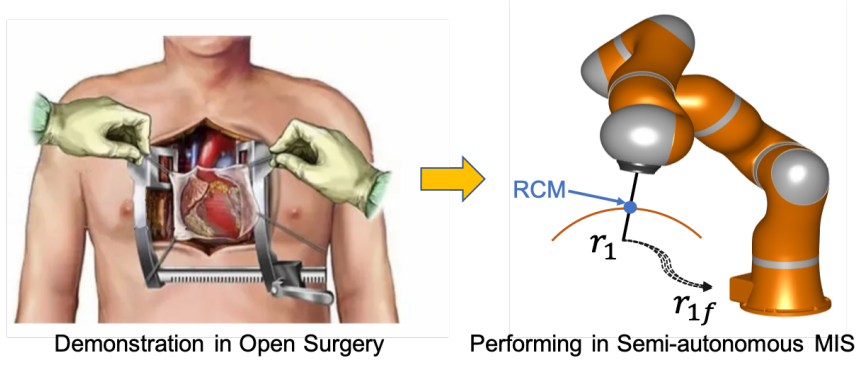

Fig. 1. Skill transfer from open surgery to MIS. The left picture depicts the multiple demonstrations operated by an experienced surgeon in open surgery while the right image explains the performing of the learned task in MIS. For RA-MIS, $\boldsymbol{r}_{1}$ is the task's initial point, and $\boldsymbol{r}_{1 f}$ is the task's final point. During the task operation, the tool must respect to the small incision on the abdominal wall.

demonstrations in this paper. The repeated surgical operation curves obtained from experienced surgeons will be adjusted. Then, to model the curves obtained from the demonstrations taught by experienced surgeons, the Gaussian Mixture Model (GMM) is utilized to model the DMP during surgical operation tasks.

1) A novel methodology of the 3-D manipulation skill modeling after multiple demonstrations is presented to the processes of surgical operation by integrating DTW and GMM based DMP.

2) Performing the learned surgical operation skills in RAMIS by utilizing a decoupled controller and respect to the RCM constraint during surgical operations simultaneously.

The developed techniques would enable the robot to learn from senior surgeons' skills, which features repetitive patterns in open surgery, such as suturing or knot tying, by using the $\mathrm{TbD}$ techniques and performing the learned surgical operations in semi-autonomous surgery in the future. The proposed approach reflects progress in comparison to the simple surgical task tracking introduced in [23] and incorporates effective TbD strategies [20] to a single controller in order to enable the robot to reproduce the demonstrated operations. Finally, the efficiency and accuracy of the proposed approach are validated with the KUKA LWR4+ robot on a 3-D printed patient's phantom.

The remainder of this paper is organized as follows. Section II contains the problem description addressed by this paper. The corresponding control methodology and control framework are presented in Section III. In Section IV, the performance results are demonstrated by using the KUKA LWR4+ robot on a patient phantom, and the conclusions of this paper are drawn in Section V.

\section{Problem Description}

Although Robot-assisted Minimally Invasive Surgery can introduce many advantages when compared with conventional methods, several critical issues need to be considered to enhance safety and improve accuracy during RAMIS. Generally, during RA-MIS surgical operations, the basic problems that should be fulfilled can be summarized as follows: movement 
constraint of the surgical tool, skills transferring method, and control system development.

Firstly, the most critical problem during the operation process of RA-MIS is safety. During surgical operations, the surgical tool should pass through the small incision on the abdominal wall of the patient's body. Meanwhile, the surgical tool should avoid making trauma to surrounding tissues and organs, which is to ensure the safety of RAMIS. For the surgical robot, this can be considered as a kinematics constraint of the surgical tool and can be achieved by the RCM constraint of the surgical robot.

Besides, how to teach the surgical robot to learn the surgical skills from an experienced surgeon is another important issue. Unlike experienced surgeons, surgical robots are not intelligent enough to make judgments and decisions autonomously during the surgical operation process. To improve surgical margins and decrease operating duration[29], surgical skills performed by an experienced surgeon in open surgery could be learned and used for semi-autonomous or autonomous operation in RAMIS [8][30]. Hence, efficient and appropriate skill learning methods need to be introduced to facilitate the learning of surgical skills by surgical robots [31], [32].

Moreover, surgical robots perform surgical operation tasks by controlling the surgical tip of the surgical robot to track the desired trajectories and further complete a series of surgical operations, such as cutting, flipping, etc. Therefore, precise control algorithms [33] and a robust robot control system [34] also should be developed to guarantee that the robot can complete the surgical tasks accurately.

\section{Methodology}

The control method discussed in this paper is intended to develop a new control approach that incorporates the TbD techniques to learn the manipulation skills involved in a specific task from multiple demonstration operations performed by an expert surgeon in open surgery. It can achieve the representation [35] of the learned motion skill in semi-autonomous MIS with a decoupled impedance controller respecting the RCM constraint.

\section{A. Modeling of the Serial Robot}

The serial manipulator's dynamic model used in this paper can be described as [36]:

$$
\boldsymbol{M}(\boldsymbol{q}) \ddot{\boldsymbol{q}}+\boldsymbol{C}(\boldsymbol{q}, \dot{\boldsymbol{q}}) \dot{\boldsymbol{q}}+\boldsymbol{g}(\boldsymbol{q})-\boldsymbol{\tau}_{e}=\boldsymbol{\tau}_{C}
$$

where $n$ is the number of the degree of freedoms (DoFs). $\boldsymbol{C}(\boldsymbol{q}, \dot{\boldsymbol{q}}) \in R^{n \times n}$ is the Coriolis force and Centrifugal matrix. $\boldsymbol{q} \in R^{n}$ is the corresponding joints value, the matrix of the corresponding inertia effects is defined as $\boldsymbol{M}(\boldsymbol{q}) \in R^{n \times n}$, and $\boldsymbol{g}(\boldsymbol{q}) \in R^{n}$ is the vector of gravity. $\boldsymbol{\tau}_{e} \in R^{n}$ and $\tau_{C} \in R^{n}$ denote the corresponding external torque and the corresponding control torques, respectively.

The formulation can be defined as follows in the task space [37][38]:

$$
\boldsymbol{M}_{X} \ddot{\boldsymbol{X}}+\boldsymbol{H}_{X} \dot{\boldsymbol{X}}+\boldsymbol{J}_{T}^{-T}(\boldsymbol{q}) \boldsymbol{g}(\boldsymbol{q})-\boldsymbol{F}_{e T}=\boldsymbol{F}
$$

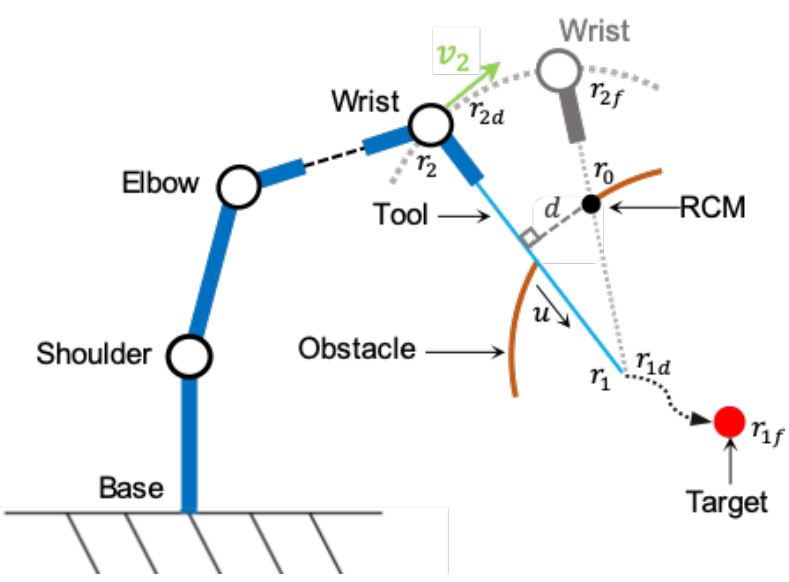

Fig. 2. RCM constraint control: a null-space kinematic controller is utilized to achieve the RCM constraint. As it is shown in the above picture, $d$ is the RCM constraint error, calculated by the distance between the trocar position $\left(\boldsymbol{r}_{0}\right)$ and the tool shaft. The tool-tip is controlled to reach the target from the actual position $\left(r_{1}\right)$ in the patient's abdomen cavity, and $\boldsymbol{v}_{2}$ is the desired velocity to drive the wrist to the desire position $\left(\boldsymbol{r}_{2 d}\right)$ until it reaches $\left(\boldsymbol{r}_{2 f}\right)$.

where the task space coordinates is $\boldsymbol{X} \in R^{m}$ and $\dot{\boldsymbol{X}} \in R^{m}$ represents the actual Cartesian velocity,

$$
\begin{aligned}
\boldsymbol{M}_{X} & =\boldsymbol{J}_{T}^{-T} \boldsymbol{M}(\boldsymbol{q}) J_{T}^{-1} \\
\boldsymbol{H}_{X} & =\boldsymbol{J}_{T}^{-T}\left[\boldsymbol{C}(\boldsymbol{q}, \dot{\boldsymbol{q}})-\boldsymbol{M}(\boldsymbol{q}) \boldsymbol{J}^{-1} \dot{\boldsymbol{J}}_{T}\right] \boldsymbol{J}_{T}^{-1} \\
\boldsymbol{F}_{e T} & =\boldsymbol{J}_{T}^{-T} \boldsymbol{\tau}_{e}
\end{aligned}
$$

In this paper, the singularity case is ignored and conclude that there is the pseudo-inverse of $\boldsymbol{J}_{T}(\boldsymbol{q}) \in R^{m \times n}$ from the base to the end-effector. The matrix $M_{X} \in R^{m \times m}$ is the Cartesian inertia. $\boldsymbol{H}_{X} \in R^{m \times m}$ is the effects of Cartesian Coriolis force and $m$ is the degrees of task space. $\boldsymbol{F}_{e T} \in R^{m}$ is the external force under the constraint condition

$$
\exists \beta \in R,\left\|\boldsymbol{F}_{e T}\right\| \leq \beta, \forall t \geq 0
$$

\section{B. Remote Center of Motion (RCM)}

To make the end-effector position $\left(\boldsymbol{r}_{1} \in R^{3}\right)$ to match the target position $\left(\boldsymbol{r}_{1 f} \in R^{3}\right)$, an interpolation technique for moving to the desired position $\left(\boldsymbol{r}_{1 d} \in R^{3}\right)$ is introduced smoothly as [39], [40]:

$$
\boldsymbol{r}_{1 d}=-k_{1}\left(\boldsymbol{r}_{1}-\boldsymbol{r}_{1 f}\right)+\dot{\boldsymbol{r}}_{1 f}
$$

where $k_{1}>0$ is a positive coefficient. Based on the $\boldsymbol{r}_{1 d}$, we can obtain the desired wrist position $\boldsymbol{r}_{1 f}$ from the admittance control model. According to the scenario with a serial robot in Fig. 2, $d$ is the distance from the RCM point $\left(\boldsymbol{r}_{0}\right)$ to the tool (the RCM constraint is enlarged for an easier understanding). The tooltip is controlled to track the target $\boldsymbol{r}_{1 d}$ from the actual position $\boldsymbol{r}_{1}$ in the patient's abdominal cavity. The value $\boldsymbol{v}_{2}$ is the velocity to move the wrist from its actual position $\boldsymbol{r}_{2}$ to its desired position $\boldsymbol{r}_{2 d}$, where the tool shaft passes through the RCM point $\boldsymbol{r}_{0}$. Hence, the final desired wrist position $\boldsymbol{r}_{2 f}$ can be obtained from:

$$
\boldsymbol{r}_{2 f}=\boldsymbol{r}_{1 d}+\frac{\boldsymbol{r}_{0}-\boldsymbol{r}_{1 d}}{\left\|\boldsymbol{r}_{0}-\boldsymbol{r}_{1 d}\right\|}\left\|\boldsymbol{r}_{2}-\boldsymbol{r}_{1}\right\|
$$


where $\boldsymbol{J}_{\boldsymbol{N}} \in R^{3 \times 7}$ is the Jacobian matrix from the robot base to the wrist, and $\boldsymbol{J}_{\boldsymbol{T}}(\boldsymbol{q})_{\boldsymbol{M}}^{+}$is the inertial-weighted pseudoinverse matrix:

$$
\boldsymbol{J}_{\boldsymbol{T}}(\boldsymbol{q})_{\boldsymbol{M}}^{+}=\boldsymbol{M}(\boldsymbol{q})^{-1} \boldsymbol{J}_{\boldsymbol{T}}(\boldsymbol{q})^{T}\left(\boldsymbol{J}_{\boldsymbol{T}}(\boldsymbol{q}) \boldsymbol{M}(\boldsymbol{q})^{-1} \boldsymbol{J}_{\boldsymbol{T}}(\boldsymbol{q})^{T}\right)^{-1}
$$

$\boldsymbol{F}_{\boldsymbol{N}}$ is the force applied on the null-space kinematics, which could be designed as:

$$
\boldsymbol{F}_{\boldsymbol{N}}=-\boldsymbol{K}_{\boldsymbol{N}}\left(\boldsymbol{r}_{2}-\boldsymbol{r}_{2 d}\right)-\boldsymbol{D}_{\boldsymbol{N}} \dot{\boldsymbol{r}}_{2 d}
$$

where $\boldsymbol{K}_{\boldsymbol{N}} \in R^{3 \times 3}$ and $\boldsymbol{D}_{\boldsymbol{N}} \in R^{3 \times 3}$ are designed null-space stiffness and damping matrices.

The final control term of the decoupling control algorithm can be expressed as follows:

$$
\tau=\tau_{T}+\tau_{N}
$$

\section{Teaching by Demonstration (TbD)}

$$
\boldsymbol{r}_{2 d}=-k_{2}\left(\boldsymbol{r}_{2}-\boldsymbol{r}_{2 f}\right)+\dot{\boldsymbol{r}}_{2 f}
$$

where $k_{2}>0$ is a positive coefficient.

\section{Decoupling Control Framework}

Then a decoupling control method [38], shown in Fig. 3, is introduced to achieve the desired tracking task on the surgical tip and guaranteeing the RCM limitation with redundant elements, separately. In this section, the proposed "Extended Jacobian method" in [41] is presented in this section to expand the operational space by formulating the end-effector and the last joint. The decoupled control architecture shown in Fig. 3 consists of two main elements[38]:

- a Cartesian compliance control strategy to track the reference trajectory $\boldsymbol{r}_{1 f}$, accounting for the representation of the learned surgical task demonstration;

- a null-space controller to drive the wrist position to $\boldsymbol{r}_{2 f}$ respecting the RCM constraint;

Next, the formulation of decoupling control will be introduced. The end velocity of the tooltip in the workspace and the joint-space angular velocity have the following form:

$$
\dot{\boldsymbol{r}}=\boldsymbol{J}_{T}(\boldsymbol{q}) \dot{\boldsymbol{q}}
$$

where $\dot{\boldsymbol{r}} \in R^{3}$ is the actual end-effector Cartesian velocity. To make the position of the end-effector $\left(\boldsymbol{r}_{1} \in R^{3}\right)$ to follow the desired trajectory $\left(\boldsymbol{r}_{1 d} \in R^{3}\right)$, the torque $\boldsymbol{\tau}_{T}$, that is, the Cartesian compliance control strategy could be designed as:

$$
\boldsymbol{\tau}_{\boldsymbol{T}}=\boldsymbol{J}_{\boldsymbol{T}}(\boldsymbol{q})^{T}\left[\boldsymbol{K}_{\boldsymbol{X}}\left(\boldsymbol{r}_{1}-\boldsymbol{r}_{1 d}\right)-\boldsymbol{D}_{\boldsymbol{X}} \dot{\boldsymbol{r}}_{1 d}\right]
$$

where $\boldsymbol{K}_{\boldsymbol{X}} \in R^{3 \times 3}$ and $\boldsymbol{D}_{\boldsymbol{X}} \in R^{3 \times 3}$ represent the diagonal stiffness and the diagonal damping matrices, respectively, which are needed to be chosen.

In addition, to drive the wrist position $\boldsymbol{r}_{2}$ to respect the RCM constraint, an additional control term, i.e., the nullspace controller, should be introduced to maintain this control objective. By utilizing the redundant degrees of freedom of the robot, the null-space controller could be defined as:

$$
\boldsymbol{\tau}_{\boldsymbol{N}}=\left(\boldsymbol{I}-\boldsymbol{J}_{\boldsymbol{T}}(\boldsymbol{q})^{T}\left(\boldsymbol{J}_{\boldsymbol{T}}(\boldsymbol{q})_{\boldsymbol{M}}^{+}\right)^{T}\right) \boldsymbol{J}_{\boldsymbol{N}}^{T} \boldsymbol{F}_{\boldsymbol{N}}
$$

An enhanced TbD control framework is introduced for modeling the demonstrated tasks in open surgery by utilizing the DTW and DMP. Recently, transferring experienced surgeons" skills to the surgical robot is attracting more and more attention in the surgical robotics area [14], [42]. To cope with such challenges, the need for developing methodology and technology in human skill transferring will reinforce to the robot-assisted surgical system.

Dynamic Time Warping (DTW) is a similarity measurement tool by extending and shortening the data length [20], [43], [44]. Except for extracting the similarity from multiple demonstrations, motor primitives of the robot manipulation should be modeled for representation. Dynamic Movement Primitive (DMP) appears to be an effective and useful way of representing the movement.

1) Preprocessing of demonstrated data using Dynamic Time Warping: To derive the similarity from the multiple demonstration curves by an experienced surgeon in open surgery, DTW has been introduced to match the different manipulation templates with varying data lengths [45]. The DTW captures flexible similarities under time distortions and features the sum of the different indices among these similarities, called Warp Path Distance, $D(i, j)$, to calculate the correlation of the two time series. For example, given the reference data series $R=\left\{r_{1}, r_{2}, r_{3}, \cdots, r_{i}, \cdots, r_{L_{1}}\right\}$ and the other demonstrated data series $T=\left\{t_{1}, t_{2}, t_{3}, \ldots, t_{j}, \ldots, t_{L_{2}}\right\}$, where $r_{i}$ and $t_{j}$ represent the values of each series, and $L_{1}$ and $L_{2}$ denote the series lengths. A distance matrix can be utilized to realign $R$ and $T$.

$$
D(i, j)=\min \left\{\begin{array}{c}
D(i, j-1) \\
D(i-1, j) \\
D(i-1, j-1)
\end{array}\right\}+d\left(t_{i}, r_{j}\right)
$$

where $i=1,2, \cdots, L_{1}, j=1,2, \cdots, L_{2}$ and $d\left(t_{i}, r_{j}\right)$ denote the distance between $r_{i}$ and $t_{j} . D\left(L_{1}, L_{2}\right)$ is the distance between $R$ and $T$ after the mapping. The best alignment can be achieved when the smallest $D\left(L_{1}, L_{2}\right)$ is obtained. The DTW is able to couple the data with different length by their similarity [46], regardless of the time sequences with a 
comparison of the general euclidean mapping. In this way, in order to realign the $R$ and $T$, DTW can be introduced to obtain a warped matrix $W=\left\{w_{1}, w_{2}, \cdots, w_{p}, \cdots, w_{P}\right\}$, where $w_{p}=\left(r_{i_{p}}, t_{j_{p}}\right), 1<p<P$ and $P$ is the warped data length. It means at the $p$ th step, $r_{i}$ and $t_{j}$ are aligned and they are saved in the warped matrix. After the processing of DTW, the aligned demonstration curves can be obtained.

2) Modeling the operation curves using discrete Dynamic Movement Primitive: Given the continuous stream of operation curves aligned with DTW, DMP [47][48] is an effective approach to identify movement primitives among them in biology motion studies. Generally, DMP consists of 2 main components: 1) a converting system to constitute the states based on dynamical structures, and 2) a canonical system $h(x)$ to generate trajectories by interpolating the factors. The detailed formula can be described as:

$$
\begin{aligned}
& \dot{x}=h(x) \\
& \ddot{y}=\alpha_{y}\left(\beta_{y}(g-y)-\dot{y}\right)+f
\end{aligned}
$$

where $y$ is the converting system states, $x$ is the canonical system states, $g$ is the end point, and

$$
f(x, g)=\frac{\sum_{i=1}^{N} \psi_{i} w_{i}}{\sum_{i=1}^{N} \psi_{i}} x\left(g-y_{0}\right)
$$

is a nonlinear function of the canonical system. $w_{i}$ is a weighting for a given basis function

$$
\psi_{i}=\exp \left(-h_{i}\left(x-c_{i}\right)^{2}\right)
$$

where $c_{i}$ and $h_{i}$ are the parameters of the Gaussian function.

\section{E. Gaussian Mixture Model}

In this paper, the GMM is applied to generate multiple patterns at the same time, which can ensure the accuracy of the action and model the uncertainty of multiple sets of demonstrating data. In the generation stage, the Gaussian Mixture Regression (GMR) and DMP are combined to model the trajectory.

Human demonstrating data generally include multiple trajectories for a single task, and these trajectories can not be exactly the same [49]. In order to learn from multiple demonstrations accurately, in our framework, GMM is needed to encode the temporal and spatial components of continuous trajectories. For learning tasks, suppose that the training dataset includes $N$ trajectories containing spatial or sensory data $\xi_{s}$, and considering the temporal component $\xi_{t}$ as an additional dimension. The dataset $\xi^{(j)}=\left\{\xi_{s}^{(j)}, \xi_{t}^{(j)}\right\}, j=$ $1, \ldots, N$ is modeled by a mixture of $K$ components, defined by probability density function:

$$
p\left(\xi^{(j)}\right)=\sum_{k=1}^{K} \alpha_{k} \mathcal{N}\left(\xi^{(j)} \mid \mu_{k}, \Sigma_{k}\right)
$$

where $\mathcal{N}\left(\xi^{(j)} \mid \mu_{k}, \Sigma_{k}\right)$ is a Gaussian conditional probability density function. GMM parameters, which can be learned by expectation-maximization (EM) algorithm, are described by $\left\{\alpha_{k}, \mu_{k}, \Sigma_{k}\right\}_{k=1}^{K}$, representing respectively prior, mean vectors and covariance matrices. After completing the training of the

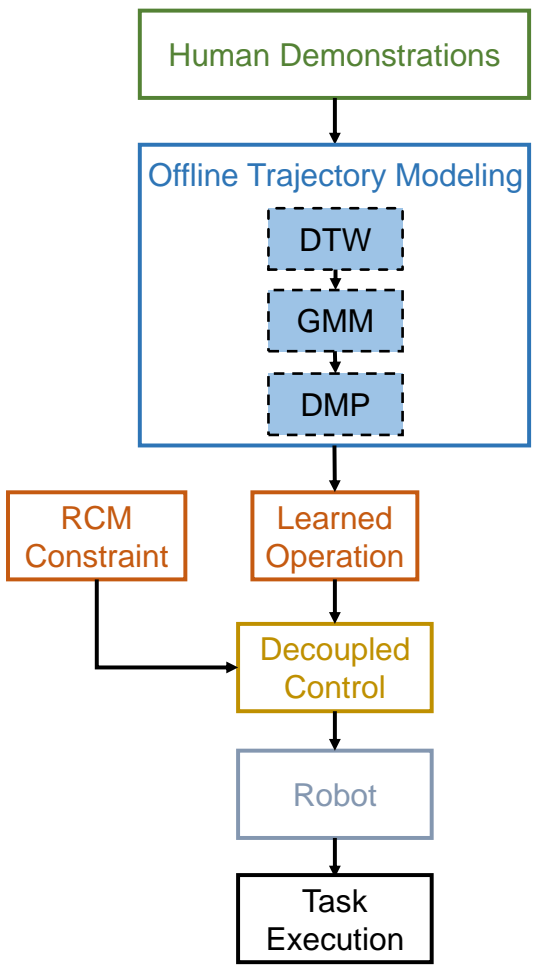

Fig. 4. Developed control framework. After multiple demonstrations, the demonstrated motion curves are aligned with DTW, and GMM is introduced to extract the similarity of the DMP for the manipulation task. A learned manipulation curve is obtained from the trained model. Finally, the learned operation is performed with the decoupled controller respecting the RCM constraint.

trajectory probability model, the next step is to generate a suitable trajectory based on the demonstrating information. Given the detailed expression of the model, GMR is employed to generate a synthesized trajectory with smaller position errors in the workspace. Based on the theorem of Gaussian conditioning, the formula of the desired trajectory can be given:

$$
\begin{aligned}
& p\left(\xi_{s} \mid \xi_{t}\right)=\sum_{k=1}^{K} \beta_{k} \mathcal{N}\left(\xi_{s} \mid \xi_{s, k}, \hat{\Sigma}_{s, k}\right) \\
& \hat{\xi}_{s, k}=\mu_{s, k}+\Sigma_{s t, k}\left(\Sigma_{t t, k}\right)^{-1}\left(\xi_{t}-\mu_{t, k}\right) \\
& \hat{\Sigma}_{s s, k}=\Sigma_{s s, k}-\Sigma_{s t, k}\left(\Sigma_{t t, k}\right)^{-1} \Sigma_{t s, k}
\end{aligned}
$$

where $\beta_{k}=p\left(k \mid \xi_{t}\right)$ is defined by the probability of the component $k$ to be responsible for $\xi_{t}$. Using the linear combination properties of Gaussian distribution, an estimation of the conditional expectation of $\xi_{s}$ given $\xi_{t}$ is thus defined by $p\left(\xi_{s} \mid \xi_{t}\right) \sim \mathcal{N}\left(\hat{\xi}_{s}, \hat{\Sigma}_{s s}\right)$ where $\hat{\xi}_{s}=\sum_{k=1}^{K} \beta_{k} \hat{\xi}_{s, k}$, $\hat{\Sigma}_{s s}=\sum_{k=1}^{K} \beta_{k}^{2} \hat{\Sigma}_{s s, k}$. Therefore, a generalized form of the motions $\hat{\xi}=\left\{\hat{\xi}_{s}, \xi_{t}\right\}$ and associated covariance matrices $\hat{\Sigma}_{s s}$ describing the constraints are computed by evaluating $\left\{\hat{\xi}_{s}, \hat{\Sigma}_{s s}\right\}$ at different time step $\xi_{t}$. Then, DMP is used to model the desired trajectory $\hat{\xi}_{s}$ for the purpose of generating 


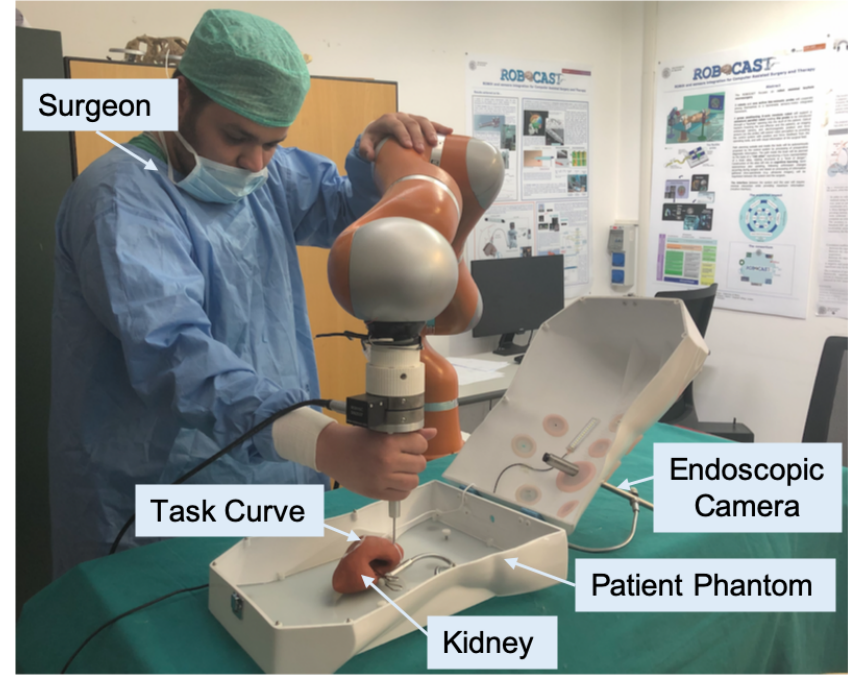

Fig. 5. Demonstration setup scene in open surgery. A kidney tissue model with a size of around $135 \times 45 \times 30 \mathrm{~mm}^{3}$ is presented in the 3-D patient phantom $\left(170 \times 210 \times 100 \mathrm{~mm}^{3}\right)$. The patient phantom is opened, and a metal clip fixes the kidney model in the abdominal cavity. A white task curve is drawn in advance along a blood vessel on the surface of the kidney to serve as the specific tracking task. The robot is activated in hands-on control mode to enable the surgeon to relocate the surgical tip by hand. The "surgeon" is commanded to do multiple demonstrations of tracking the white task curve with the surgical tip.

generalized trajectories under different target positions.

\section{F. Control Framework Development}

The control framework integrates the procedures of teaching by demonstration and decoupled control with the RCM constraint, and it is depicted in Fig. 4, which contains the following steps.

(1) Firstly, the human operator guides the robot to perform the demonstration task a couple of times through kinesthetic teaching, and the corresponding operation curves are recorded [50];

(2) Since the performing time is varying, the technique of DTW is adopted for aligning the curves with the same data length. Through the alignment processing, not only can the trajectories' length in the input training data be guaranted to be the same and aligned in time, but also the data at the same time step can be modeled together;

(3) At the same time, the GMM is used to model multiple trajectories, and GMR is used to generate one trajectory from the multiple curves;

(4) Finally, a learning curve is derived from the trained DMP, and it is performed using a robot under the RCM constraint through a decoupled controller.

The performance of the proposed approach is tested in a lab setup environment using a 7 DoFs serial robot. The ability to learn from multiple demonstrations and to perform the learned manipulation skill in semi-autonomous MIS in demonstrated and discussed in the following section.
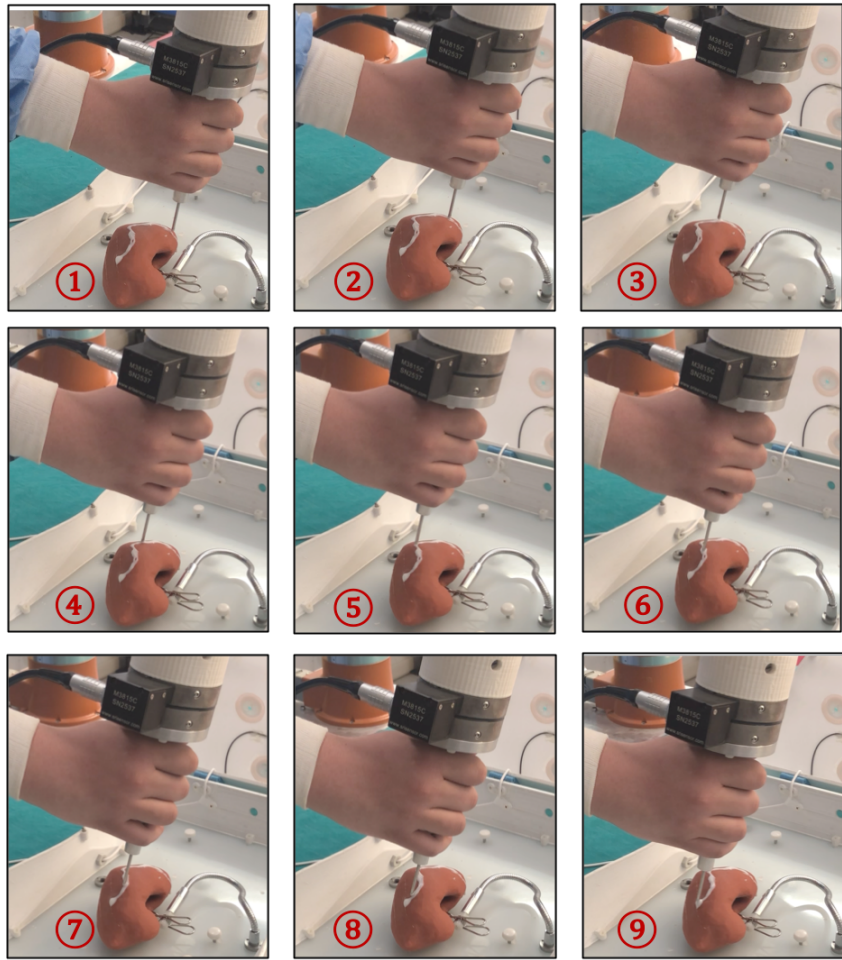

Fig. 6. Demonstration task tracking procedure. The numbers (1-9) indicate the tracking procedure by hands-on demonstration in open surgery. The 1st picture shows the starting point of the tracking tasks, and the 9th picture represents the corresponding final point. The "surgeon" use hands to hold on the tool shaft and move the tool tip following the white task curve on the kidney.

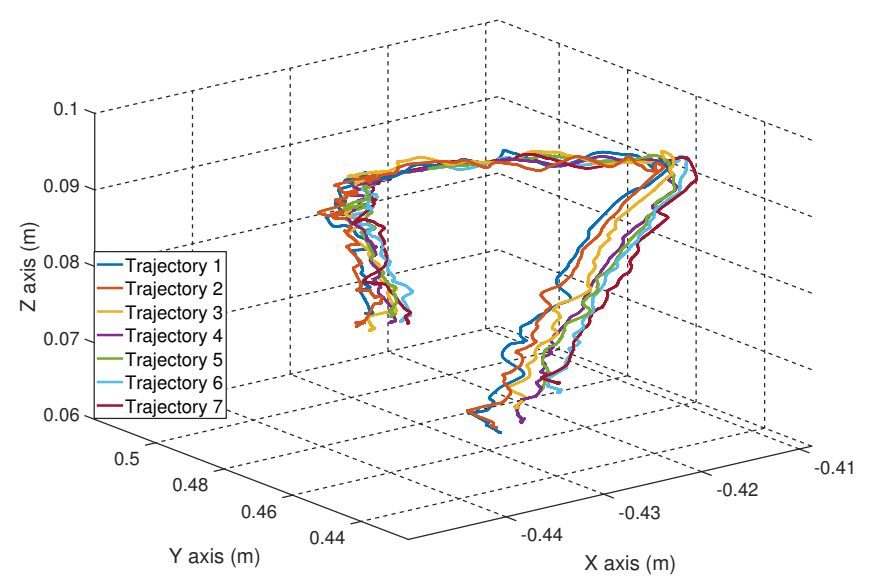

Fig. 7. Demonstrated operation curves in 3-D. The hands-on demonstrations are repeated 7 times in open surgery.

\section{EXPERIMENTAL DEMONSTRATION AND RESUlts}

An overview of the developed experimental surgical system is shown in Fig. 5. A redundant robot (LWR4+, KUKA, Germany) serves as the serial robot torque-controller through Fast Research Interface (FRI), which provides direct lowlevel real-time access to the robot controller (KRC) at rates of $500 \mathrm{~Hz}$. An human operator (namely the surgeon) would manually operate the robot the generate the demonstrating data. The RCM constraint is provided by a 3-D printed surgery 

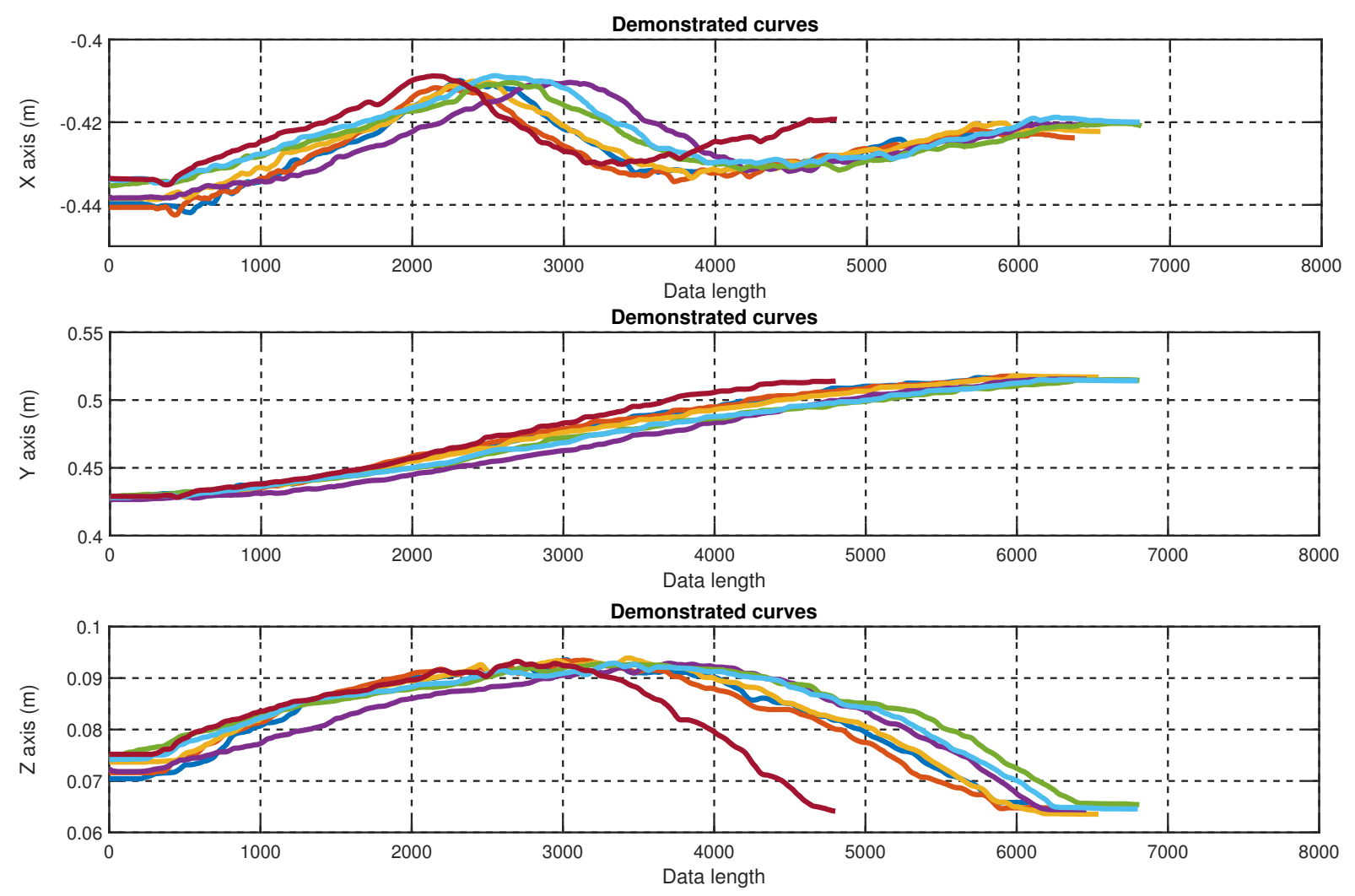

Fig. 8. Demonstrated operation curves in 3-axis. The demonstrated data lengths are different due to the difference of the operation time.
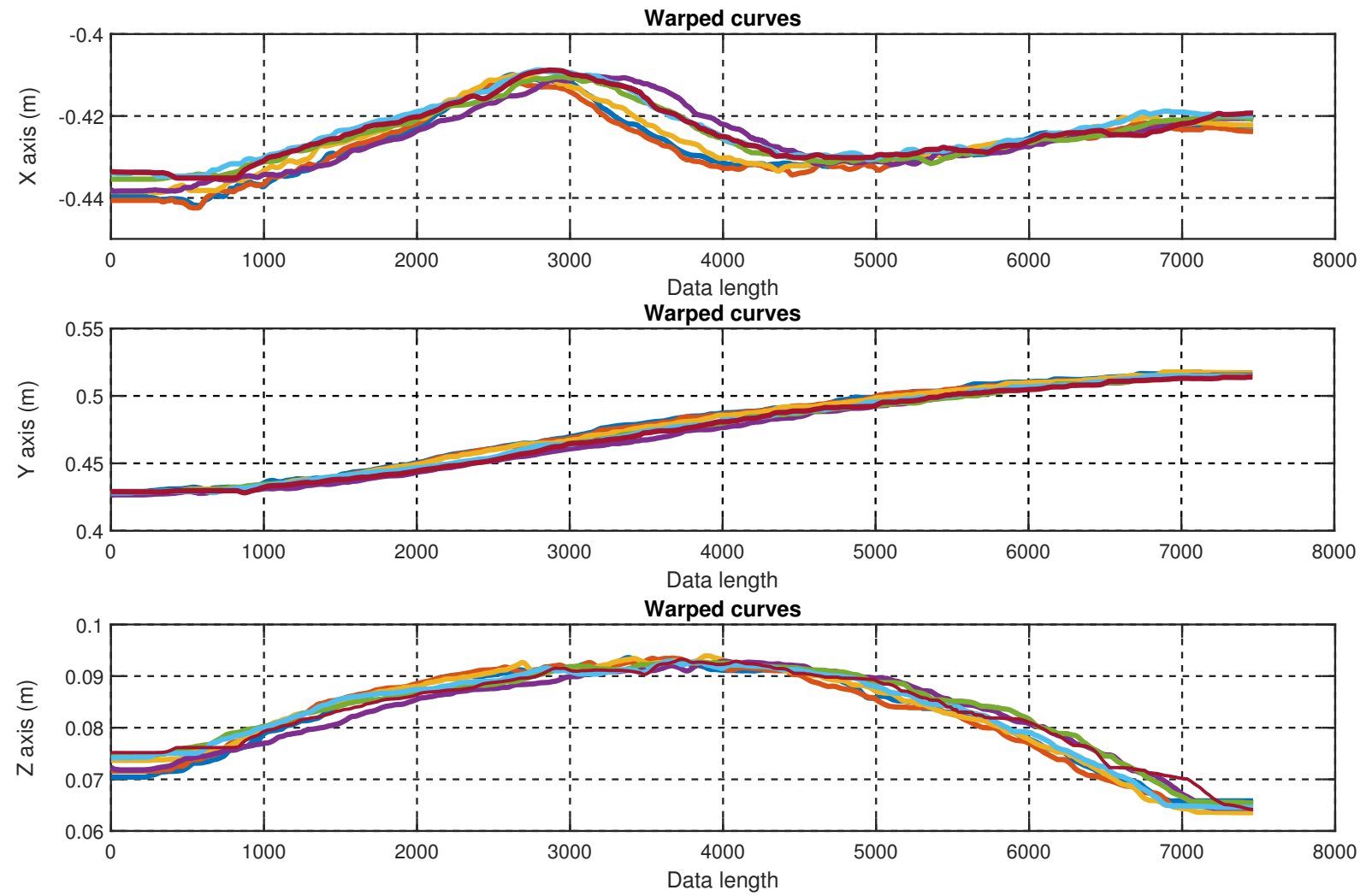

Fig. 9. Warped operation curves in 3-axis. The curves are warped with DTW to align the data. 
human phantom. The size of the phantom is similar to the real human abdomen and the distribution of the incisions is same as the real surgery. Each small incision is equipped with a silicone material to simulate the physical interaction. In this section, in order to evaluate the performance of the proposed method, some experiments are carried out. The experimental procedures mainly include as follows:

1) Firstly, the desired trajectory is generated through operating the robot arm multiple times in surgery environment by human operators, the GMM-based DMP presented in Section III is used to generate a learned trajectory.

2) Then, the KUKA robot is performed to move along the learned trajectory to accomplish the surgical operation with RCM constraint in the autonomous MIS.

\section{A. Teaching by Demonstration in open surgery}

In order to evaluate the proposed approach, an experiment using the KUKA robot is performed to demonstrate its feasibility. The scenario of hands-on demonstration in open surgery is shown in Fig. 5. A 3-D printed patient phantom and a kidney organ in the abdomen cavity are used for demonstration. A white curve is drawn on the kidney to serve as a demonstration task.

First of all, the patient phantom is opened, and a subject is asked to move the robot by hands-on control to track the demonstration task, as shown in Fig. 6. The surgeon manipulates the robotic arm to track the desired trajectory with the sequence steps. Particularly, the demonstration task is repeated 7 times. The corresponding 3-D demonstration curves of the tracking task are shown in Fig. 7. As shown in Fig. 8, since it is impossible to ask a surgeon to perform a task precisely multiple times, these trajectories are quiet different from each other, especially the data length. In other words, in the process of modeling multiple trajectories by GMM, there remains a problem concerning the robustness of the model to the temporal variability across the demonstrated movements. Thus, it could be useful to align the different demonstrations automatically before further processing. As it is shown in Fig. 8, the data length of the curves are different. To model the manipulation skills, it is essential to align the operation curves and then extract the similarity of the curves by using DMP. Hence the DTW is employed to warp the curves according to the time sequences from 1 to 9 . After the pre-processing, the operation curves are aligned according to each axis, as shown in Fig. 9. Through the alignment processing, not only can the trajectories' length in the input training data be guaranteed to be the same and aligned in time, but also the data at the same time step can be modeled together. Then GMM is introduced to model the DMP of the operation curve, and a learned 3-D is derived from the trained DMP. Finally, the learned manipulation motion is derived from multiple demonstrations. Fig. 10 shows the learned 3-D task by teaching by demonstration. Thus, using the proposed algorithm, the approach could learn the surgical operation skills of DMP with multiple demonstrations of a specific task.

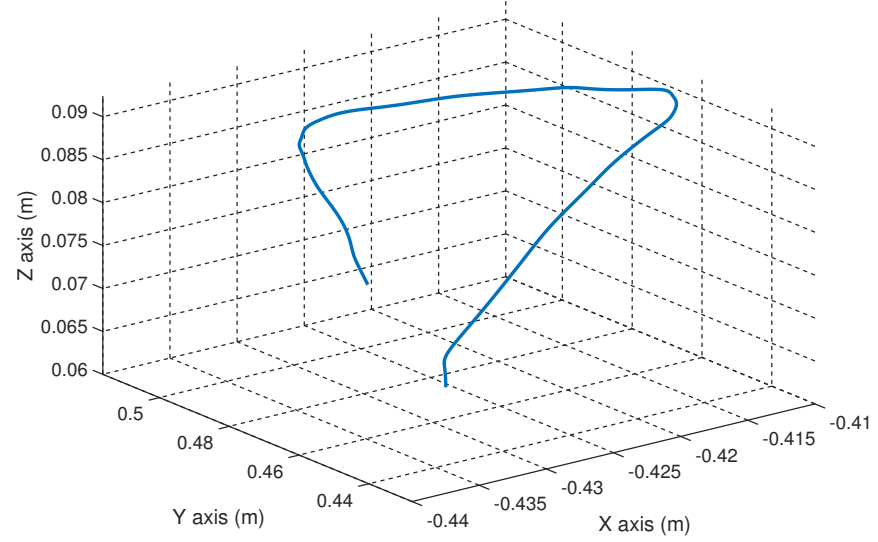

Fig. 10. Learned operation curves in 3-D

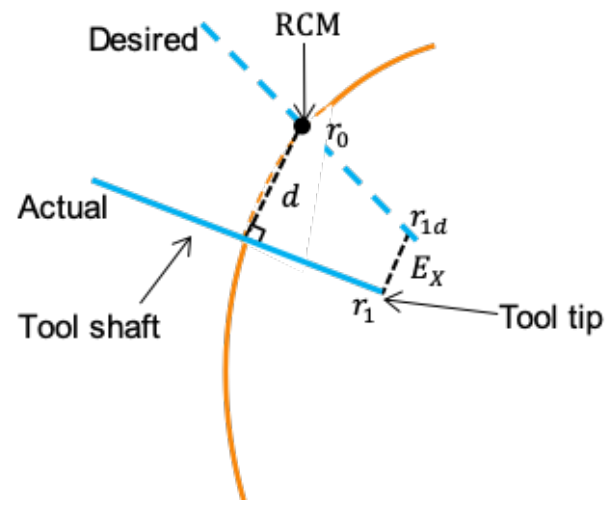

Fig. 12. Performance measurement. $d$ is the RCM constraint error and $E_{X}$ is the Cartesian error on the tool tip. The "Actual" link means the actual tool shaft placement while the "Desired" link represents its corresponding desired placement.

\section{B. Performing learned trajectory in semi-autonomous MIS}

After teaching by demonstration, the learned trajectory is performed in semi-autonomous surgery, which includes two procedures shown in Fig. 11. The left picture of Fig. 11 shows the first procedure to use hands-on control to locate the position of the RCM constraint. Then, surgical tool inserted to inside the abdominal cavity to reach the initial point of the task. The right image shows the second step to perform 
(a) Cartesian position error

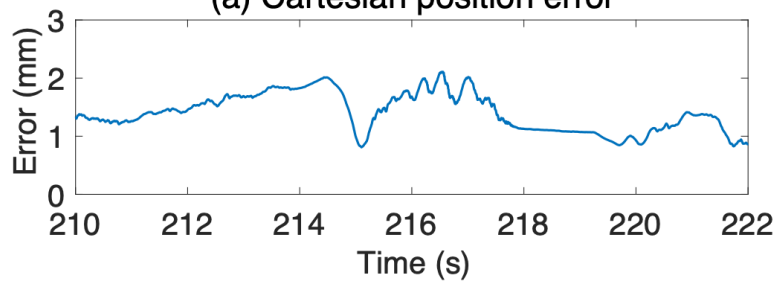

(b) RCM constraint error

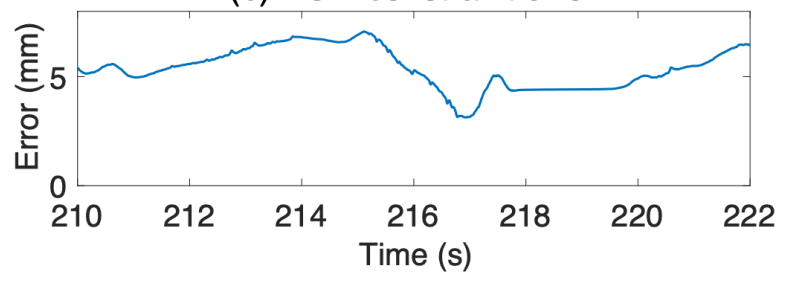

Fig. 13. Online performance for performing the learned trajectory

the learned manipulation under surgeon's supervision from the visual interface.

It should be noticed that the hands-on control is activated in the first procedure and the decoupled controller is used to perform the learned trajectory respecting the RCM constraint during the second procedure. As it is shown in Fig. 11, to locate the RCM constraint, the subject uses hands-on control. Then the subject inserts the surgical instrument into the patient abdomen phantom via the small incisions, and the robot autonomously tracks the desired trajectory to perform the surgical task by respecting the RCM constraint. During the autonomous tracking, the surgeon supervises the procedure from the visual interface and holds the emergency button for safety issue.

The decoupled impedance parameters of the controller can be found in our previous works [39]. The performance is measured according to Fig. 12 throughout the implementation of the learned path. It depicts the error of Cartesian position, $\boldsymbol{E}_{r}$, and the error of the RCM boundary, $d$, which are calculated as follows:

$$
\begin{aligned}
& \left\|\boldsymbol{E}_{r}\right\|=\left\|r_{1 d}-r_{1}\right\| \\
& d=\left\|\left(r_{0}-r_{1}\right) \times \hat{u}\right\|
\end{aligned}
$$

where $\hat{u}$ is the direction vector of the tool shaft.

Fig. 13 shows the online performance while executing the learned task with an RCM constraint. It shows that the robot can perform the learned trajectories with taking account the RCM constraint. The RCM constraint error and the Cartesian position error is constrained in a small area within $2 \mathrm{~mm}$ and $6 \mathrm{~mm}$, respectively.

\section{CONCLUSION}

This paper proposes an approach to introduce Teaching by Demonstration (TbD) techniques for Robot-Assisted Minimally Invasive Surgery (RA-MIS), where an RCM kinematic constraint is presented. It aims at using the benefits of ease manipulation in open surgery and smaller incision in MIS. More specifically, the approach consists of i) DTW to measure the similarity among the multiple demonstrations, ii)
GMM based DMP to model the motion primitives of human demonstrations, and iii) a hierarchical control framework to perform the learned surgical task respecting RCM constraint. The methods of DTW and DMP are utilized to analyze and generalize the learned operations curves demonstrated by the surgeon in open surgery. Then the learned motion is represented in semi-autonomous MIS using a decoupled control methodology. The experimental demonstration is performed on 3-D printed patient phantom by using KUKA LWR4+ to validate the quality of the proposed method. The findings show that the introduced control algorithm not only is able to learn the human operation skill from multiple demonstrations on a specific task but also can transfer the learned motion from open surgery to MIS by guaranteeing the RCM constraint.

These preliminary results demonstrate that skill transfer from open surgery to MIS is feasible. The learning ability and RCM constraints are guaranteed. Future works will exploit the developed control approach to enable the robot to learn more complex senior surgeons' skills, such as actual suturing or knot tying with organ models. Additionally, camera movement, while a surgical task can also be achieved following this approach. By the way, skill transferring in this paper only takes into account the motion skills regardless of the stiffness during the operation. Hence, the interaction force of the demonstrated task will be integrated, and the autonomous camera will be considered to achieve accurate tracking in future works.

\section{REFERENCES}


[11] B. Zhong, J. Cao, A. McDaid, S. Q. Xie, and M. Zhang, "Synchronous position and compliance regulation on a bi-joint gait exoskeleton driven by pneumatic muscles," IEEE Transactions on Automation Science and Engineering, 2020.

[12] J.-R. Li, J.-L. Fu, S.-C. Wu, and Q.-H. Wang, "An active and passive combined gravity compensation approach for a hybrid force feedback device," Proceedings of the Institution of Mechanical Engineers, Part C: Journal of Mechanical Engineering Science, p. 0954406220974052.

[13] Z. Li, B. Huang, A. Ajoudani, C. Yang, C.-Y. Su, and A. Bicchi, "Asymmetric bimanual control of dual-arm exoskeletons for humancooperative manipulations," IEEE Transactions on Robotics, vol. 34, no. 1, pp. 264-271, 2017.

[14] S. Calinon, D. Bruno, M. S. Malekzadeh, T. Nanayakkara, and D. G. Caldwell, "Human-robot skills transfer interfaces for a flexible surgical robot," Computer methods and programs in biomedicine, vol. 116, no. 2, pp. 81-96, 2014.

[15] C. Yang, C. Zeng, P. Liang, Z. Li, R. Li, and C.-Y. Su, "Interface design of a physical human-robot interaction system for human impedance adaptive skill transfer," IEEE Transactions on Automation Science and Engineering, vol. 15, no. 1, pp. 329-340, 2017.

[16] T. Osa, N. Sugita, and M. Mitsuishi, "Online trajectory planning and force control for automation of surgical tasks," IEEE Transactions on Automation Science and Engineering, vol. 15, no. 2, pp. 675-691, 2017.

[17] F. Petitjean, A. Ketterlin, and P. Gançarski, "A global averaging method for dynamic time warping, with applications to clustering," Pattern Recognition, vol. 44, no. 3, pp. 678-693, 2011.

[18] P. Kormushev, S. Calinon, and D. G. Caldwell, "Imitation learning of positional and force skills demonstrated via kinesthetic teaching and haptic input," Advanced Robotics, vol. 25, no. 5, pp. 581-603, 2011.

[19] C. Yang, C. Zeng, Y. Cong, N. Wang, and M. Wang, "A learning framework of adaptive manipulative skills from human to robot," IEEE Transactions on Industrial Informatics, vol. 15, no. 2, pp. 1153-1161, 2018.

[20] C. Li, C. Yang, Z. Ju, and A. S. Annamalai, "An enhanced teaching interface for a robot using dmp and gmr," International journal of intelligent robotics and applications, vol. 2, no. 1, pp. 110-121, 2018.

[21] H. Sadeghian, F. Zokaei, and S. H. Jazi, "Constrained kinematic control in minimally invasive robotic surgery subject to remote center of motion constraint," Journal of Intelligent \& Robotic Systems, vol. 95, no. 3-4, pp. 901-913, 2019.

[22] Y. Lin, H. Min, H. Zhou, and F. Pei, "A human-robot-environment interactive reasoning mechanism for object sorting robot," IEEE Transactions on Cognitive and Developmental Systems, vol. 10, no. 3, pp. 611-623, 2017.

[23] H. Su, Y. Hu, H. R. Karimi, A. Knoll, G. Ferrigno, and E. De Momi, "Improved recurrent neural network-based manipulator control with remote center of motion constraints: Experimental results," Neural Networks, vol. 131, pp. 291-299, 2020.

[24] H. Su, W. Qi, C. Yang, J. Sandoval, G. Ferrigno, and E. De Momi, "Deep neural network approach in robot tool dynamics identification for bilateral teleoperation," IEEE Robotics and Automation Letters, vol. 5, no. 2, pp. 2943-2949, 2020.

[25] H. Su, J. Sandoval, P. Vieyres, G. Poisson, G. Ferrigno, and E. De Momi, "Safety-enhanced collaborative framework for tele-operated minimally invasive surgery using a 7-dof torque-controlled robot," International Journal of Control, Automation and Systems, vol. 16, no. 6, pp. 29152923, 2018.

[26] M. Zhang, S. Q. Xie, X. Li, G. Zhu, W. Meng, X. Huang, and A. J. Veale, "Adaptive patient-cooperative control of a compliant ankle rehabilitation robot (carr) with enhanced training safety," IEEE Transactions on Industrial Electronics, vol. 65, no. 2, pp. 1398-1407, 2017.

[27] Z. Li, J. Li, S. Zhao, Y. Yuan, Y. Kang, and C. P. Chen, "Adaptive neural control of a kinematically redundant exoskeleton robot using brain-machine interfaces," IEEE transactions on neural networks and learning systems, vol. 30, no. 12, pp. 3558-3571, 2018.

[28] H. Su, W. Qi, Y. Hu, H. R. Karimi, G. Ferrigno, and E. De Momi, "An incremental learning framework for human-like redundancy optimization of anthropomorphic manipulators," IEEE Transactions on Industrial Informatics, 2020.

[29] A. Attanasio, B. Scaglioni, M. Leonetti, A. F. Frangi, W. Cross, C. S. Biyani, and P. Valdastri, "Autonomous tissue retraction in robotic assisted minimally invasive surgery-a feasibility study," IEEE Robotics and Automation Letters, vol. 5, no. 4, pp. 6528-6535, 2020.

[30] T. Liu and M. C. Cavusoglu, "Needle grasp and entry port selection for automatic execution of suturing tasks in robotic minimally invasive surgery," IEEE Transactions on Automation Science and Engineering, vol. 13, no. 2, pp. 552-563, 2016. 


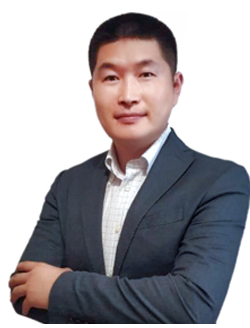

HANG SU (Member, IEEE) received the M.Sc. degree in control theory and control engineering in South China University of Technology, Guangzhou, China, in 2015 and the Ph.D. degree in Bioengineering from Politecnico di Milano, Milano, Italy, in 2019. He participated in the EU funded project (SMARTsurg) in the field of Surgical Robotics. Dr. Hang Su is currently working in the Department of Electronics, Information and Bioengineering (DEIB) of Politecnico Di Milano. He is currently a Program Chair of IEEE International Conference on Advanced Robotics and Mechatronics (ICARM 2021). He also severs as the Associate Editor for the IEEE International Conference on Robotics and Automation (ICRA) and the IEEE/RSJ International Conference on Intelligent Robots and Systems (IROS), the IEEE International Conference on Robot and Human Interactive Communication (Ro-man) and the IEEE International Conference on Advanced Robotics and Mechatronics (ICARM), and the Guest Associate Editor for a couple of journals, like IEEE Robotics and Automation Letters, Complexity, Actuators, Mathematical Problems in Engineering, Sensors, Frontiers in Robotics and AI, etc..

He was a recipient of the ICRA 2019 Travel Award funded by the IEEE Robotics and Automation Society. His main research interests include control and instrumentation in medical robotics, human-robot interaction, surgical robotics, deep learning, bilateral teleoperation, etc..

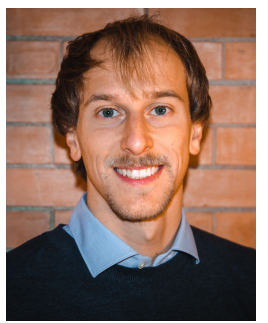

ANDREA MARIANI received his M.Sc. degree cum laude in Biomedical Engineering in Politecnico di Milano (Italy), in 2018. He also achieved the Alta Scuola Politecnica diploma cum laude in 2018, a double-degree program for 150 young talents selected among the students from Politecnico di Milano and Torino. He is currently a PhD student in BioRobotics at Scuola Superiore Sant'Anna (Pisa, Italy) and he is working in the field of medical robotics. In particular, his research focuses on simulation-based training for robot assisted surgery and high intensity ultrasound therapeutic technologies.

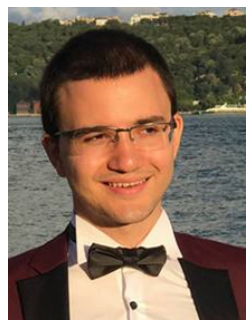

SALIH ERTUG OVUR received his double B.Sc. degree in Mechanical Engineering and Automation and Control Engineering in Istanbul Technical University (Turkey) with best student awards from both degrees and recently graduated from M.Sc. in Automation and Control Engineering at Politecnico di Milano (Italy) with a full score. He is currently working as research collaborator at NEARLAB and his research focuses on sensor fusion, humanrobot/computer interaction, control and instrumentation, and AR-VR applications.

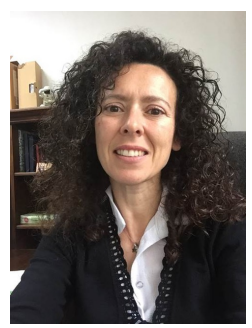

ARIANNA MENCIASSI (Senior Member, IEEE) received the M.S. degree in physics from the University of Pisa, Italy, in 1995, and the Ph.D. degree in bioengineering from the Scuola Superiore Sant'Anna of Pisa, in 1999. She is currently a Full Professor with the BioRobotics Institute, Scuola Superiore Sant'Anna, and she is the Area Leader of the Surgical Robotics and Allied Technologies Group. Her main research interests include biomedical robotics, surgical robotics, microsystem technology, nanotechnology, with a special attention to the synergy between robot-assisted therapy and micro/nanotechnology-related solutions. Prof. Menciassi served in the Editorial Board of the IEEE-ASME Transaction on Mechatronics. She is now Topic Editor in Medical Robotics for the International Journal of Advanced Robotic Systems, Associate Editor of the IEEE Transactions on Medical Robotics and Bionics, and Editorial Board Member of the APL Bioengineering. She serves also as Co-Chair of the IEEE-RAS Technical Committee on Surgical Robotics.

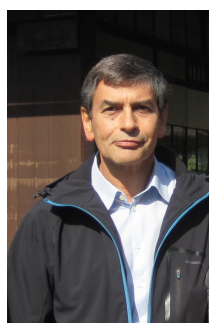

GIANCARLO FERRIGNO (Senior Member, IEEE) received the M.Sc. degree in electrical engineering and the Ph.D. degree in bioengineering from the Politecnico di Milano, Milan, Italy.

$\mathrm{He}$ is the Founder of the Neuroengineering and Medical Robotics Laboratory with the Department of Electronics, Information and Bioengineering, Politecnico di Milano, in 2008, and a Lecturer of Medical Robotics. He is a Full Professor with the Politecnico di Milano. He has been the European Coordinator of three FP7 EU projects on ICT. Two of them, ROBOCAST (STREP 2008-2010) and ACTIVE (Integrated project 2011-2015) are in the field of the Surgical Robotics. MUNDUS (STREP 2010-2013) is in the field of Assistive and Rehabilitative Robotics. He has co-authored 20 papers (ISI Web of Knowledge) in the robotic field from 2011 to 2014. Dr. Ferrigno is working in the JWG9 ISO standard group for Surgical Robots collateral standard and organized several workshops in the surgical robotics for the last three years.

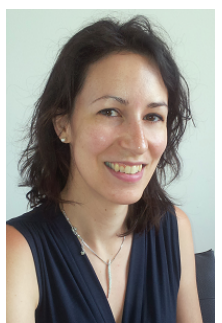

ELENA DE MOMI (Senior Member, IEEE) received the M.Sc. and Ph.D. degrees in biomedical engineering from the Politecnico di Milano, Milan, Italy, in 2002 and 2006, respectively.

She is an Associate Professor with the Department of Electronics, Information and Bioengineering, Politecnico di Milano. She is co-founder of the Neuroengineering and Medical Robotics Laboratory, in 2008, being responsible of the Medical Robotics section. IEEE Senior Member, she is currently Associate Editor of the Journal of Medical Robotics Research, of the International Journal of Advanced Robotic Systems, Frontiers in Robotics and AI and Medical \& Biological Engineering \& Computing. From 2016 she has been an Associated Editor of IEEE ICRA, IROS and BioRob and she is currently Publication Co-Chair of ICRA 2019. She is responsible for the lab course in Medical Robotics and of the course on Clinical Technology Assessment of the MSc degree in Biom. Eng. at Politecnico di Milano and she serves in the board committee of the $\mathrm{PhD}$ course in Bioengineering. 